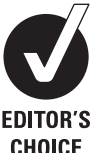

CHOICE

\title{
A qualitative approach to Bayes' theorem
}

\section{Mitchell A Medow, ${ }^{1}$ Catherine R Lucey ${ }^{2}$}

10.1136/ebm-2011-0007

${ }^{1}$ Section of General Internal Medicine, Boston University School of Medicine, Boston, Massachusetts, USA ${ }^{2}$ Internal Medicine, The Ohio State University College of Medicine, Columbus, Ohio, USA

\section{Correspondence to:}

Dr Mitchell A Medow

Section of General Internal Medicine, Boston University

School of Medicine, Crosstown

Center Building, 2nd Floor, 801

Massachusetts Avenue, Boston,

MA 02118, USA;

mitchell.medow@bmc.org

Accepted 5 July 2011

\section{Abstract}

While decisions made according to Bayes' theorem are the academic normative standard, the theorem is rarely used explicitly in clinical practice. Yet the principles can be followed without intimidating mathematics. To do so, one can first categorise the prior-probability of the disease being tested for as very unlikely (less likely than $10 \%)$, unlikely (10-33\%), uncertain (34-66\%), likely (67-90\%) or very likely (more likely than $90 \%)$. Usually, for disorders that are very unlikely or very likely, no further testing is needed. If the prior probability is unlikely, uncertain or likely, a test and a Bayesian-inspired update process incorporating the result can help. A positive result of a good test increases the probability of the disorder by one likelihood category (eg, from uncertain to likely) and a negative test decreases the probability by one category. If testing is needed to escape the extremes of likelihood (eg, a very unlikely but particularly dangerous condition or in the circumstance of population screening, or a very likely condition with a particularly noxious treatment), two tests may be needed to achieve. Negative results of tests with sensitivity $\geq 99 \%$ are sufficient to rule-out a diagnosis; positive results of tests with specificity $\geq 99 \%$ are sufficient to rule-in a diagnosis. This method overcomes some common heuristic errors: ignoring the base rate, probability adjustment errors and order effects. The simplicity of the method, while still adhering to the basic principles of Bayes' theorem, has the potential to increase its application in clinical practice.

Bayes' theorem ${ }^{1}$ remains the normative standard for diagnosis, but it is often violated in clinical practice. Attempts to simplify its application with diagnostic computer programs, ${ }^{23}$ nomograms, ${ }^{4}$ rulers ${ }^{5}$ or internet calculators ${ }^{6}$ have not helped to increase its use. Bayes' theorem helps overcome many well-known cognitive errors in diagnosis, such as ignoring the base rate, probability adjustment errors (conservatism, anchoring and adjustment) and order effects. ${ }^{7}$ Bayes' theorem and its underlying precepts are introduced early in medical school and medical texts, for example, Chapter 3 of 392 chapters in Harrison's Principles of Internal Medicine. ${ }^{8}$ Even so, adherence to Bayes' principles is all but absent - low probability diseases are still tested for causing unneeded cost and risk, and high probability diseases are ignored when a single negative test returns.

The basic idea of Bayes' theorem for medical diagnosis is well accepted. A diagnosis is not necessarily confirmed just because a test was positive. Diagnosis is usually not a binary decision (ie, true of false) turning on a single datum, but a dynamic probabilistic assessment. The post-test probability (also called the updated probability, posterior-probability or positive-predictive value) of a diagnosis is dependent on how likely the diagnosis was before the test was done (the pretest probability, also referred to as the prevalence or prior-probability), the test result (positive or negative) and the ability of the test to discriminate between those afflicted and not afflicted with the disease (test characteristics expressed as sensitivity and specificity, or likelihood ratios). A simple formula, Bayes' theorem, combines these elements to produce the post-test probability of the disease. A positive test increases confidence in a diagnosis, but usually does not indicate certainty. Whether this confidence exceeds a treatment (or action) threshold ${ }^{9}$ remains a decision for the clinician and patient. Likewise, a negative test decreases confidence in a diagnosis, but rarely rules it out completely. It is up to those involved to decide if further action is warranted.

What if an easy, non-mathematical method to apply these concepts were available? Could the application of Bayes' theorem find its appropriate place in clinical practice and not be relegated to academic exercises for medical students and residents? Could its benefits in clinical practice finally be realised? There is a simple, qualitative or categorical application of Bayes' theorem that might ease the application of Bayes' underlying precepts. The method is based on categorising the pretest probability and handling a small set of probabilistic categories instead of the full spectrum of continuous probabilities, thus eliminating the need for mathematical calculations. In this paper, we first introduce this qualitative method. Then we present the mathematical justification for the method and the conditions under which it holds. Finally we present some special cases that reinforce the method.

\section{Qualitative Bayes' theorem}

Bayes' theorem's concepts can be applied using qualitative methods. First one must commit to the pretest probability - how likely the diagnosis is from the start. This probability is expressed categorically - very unlikely (less likely than 10\%), unlikely (between 10\% and 33\%), uncertain (between 34\% and 66\%), likely (between 67\% and 90\%) or very likely (more likely than 90\%) (table 1).

If the initial assessment is very unlikely or very likely, then in most cases it is not worth further testing according to Bayes' theorem - the results would either confirm what is already near certain or it would minimally move the post-test probability in the opposite direction. Either way, the clinician would not normally take additional actions. There are at least two situations where it is still important to proceed with further testing. First, if the diagnosis is very unlikely but needs to be ruled out with more certainty, as in a very dangerous disease, the clinician may want to proceed with testing. For example, at what probability is the clinician willing to send a patient home in whom a diagnosis of subarachnoid haemorrhage is being considered? Similarly, if the diagnosis is very likely, but 
Table 1 Categorical probabilities

\begin{tabular}{ll}
\hline Categorical probability & Numerical probability \\
\hline Very unlikely & Less likely than $10 \%$ \\
Unlikely & Between $10 \%$ and $33 \%$ \\
Uncertain & Between $34 \%$ and $66 \%$ \\
Likely & Between $67 \%$ and $90 \%$ \\
Very likely & More likely than $90 \%$ \\
\hline
\end{tabular}

needs to be ruled in with more certainty, such as when the treatment is especially dangerous or noxious, further testing is indicated. For example, at what probability is the clinician willing to commit a patient with liver disease to a course of anticoagulation with warfarin for a deep venous thrombosis?

If the probability is in one of the intermediate categories, then further testing is appropriate. The clinician may order a test and then interpret the results. A positive result moves the clinician to the next more likely category. A negative result moves the clinician to the next less likely category.

For example, if the clinician is seeing a 35-year-old man in the office who presents with substernal, exertional chest pain that was relieved with rest, the patient has anginal chest pain. His pretest probability of having coronary artery disease (CAD) is likely, about $70 \% .^{10}$ Further testing is warranted and a stress test is ordered. If the result is negative, the diagnosis of CAD is uncertain not absent. If the result is positive, CAD is very likely. But, if the patient were a woman, her pretest probability of having CAD is unlikely (about 26\%). Further testing is also warranted. In this circumstance, if the stress test is negative, the diagnosis of CAD is very unlikely. If the test is positive, CAD is uncertain - not definitively present.

\section{When does the qualitative approach to} applying Bayes' theorem work?

Using the above intuitive cut-offs, and tests with sensitivities and specificities between $80 \%$ and $90 \%$, the above procedure is a good approximation to Bayes' theorem.

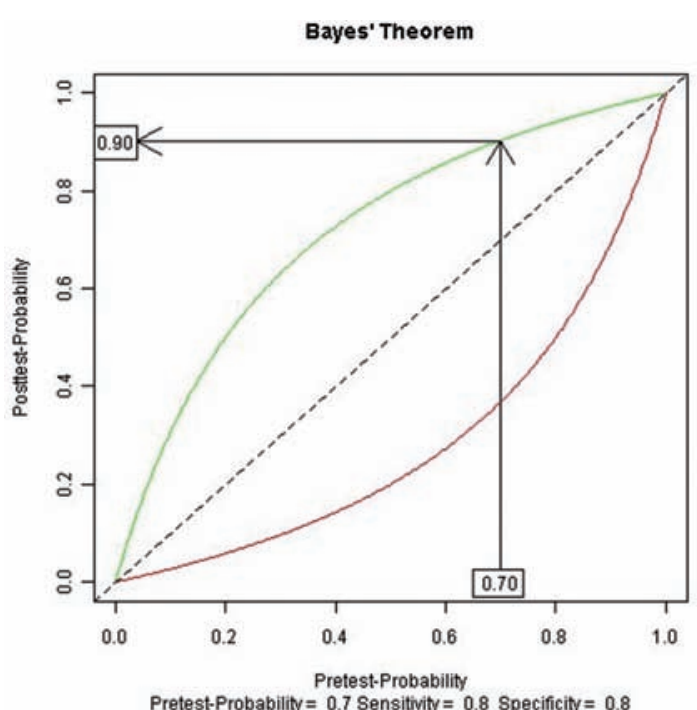

Figure 1 Graphical interpretation of Bayes' theorem.
A graphical approach to Bayes' theorem can demonstrate how the qualitative approximation works (figure 1). Here the horizontal-axis is the pretest probability, the curves represent the relationship between the pretest probability and the post-test probability for a given sensitivity and specificity (80\% for each in this example, roughly corresponding to the test characteristics for a nuclear stress test) and the vertical-axis is the post-test probability. The diagonal line is usually included and represents no change in the post-test probability with the test result (ie, the test did not change the clinician's assessment of the probability). Separate curves represent a positive result (green), which increases the post-test probability (ie, is above the diagonal line), and a negative result (red), which reduces the post-test probability. To use Bayes' theorem, one starts on the horizontal-axis at the appropriate pretest probability and draws a vertical line until it intersects the appropriate curve for a positive (green) or negative test (red) result. One then draws a horizontal line to find the appropriate post-test probability on the vertical-axis. Figure 1 exemplifies this for the case above, a man with anginal chest pain and a positive stress test. One first locates 70\% on the horizontal-axis, follows the arrow up until it intersects the positive result (green) curve, then follows the arrow horizontally until it intersects the vertical-axis at the post-test probability of $90 \%$. A similar procedure is followed for a negative test, using the red line, giving a post-test probability of $37 \%$.

In the categorical case, all the pretest probabilities between 67\% and 90\% (the likely category) need to be considered while holding the sensitivity and specificity constant at $80 \%$. This only needs to be done for the lower $(67 \%)$ and the upper limits (90\%), see figure 2 . For a positive test, the post-test probabilities range between $89 \%$ and 97\% (arrows). To get the lower limit of post-test probability for a positive test, one follows the arrow from a pretest probability of 67\% up until point A in figure 2 (where the arrow intersects the curve representing Bayes' theorem's post-test probability for a positive result), and then reads

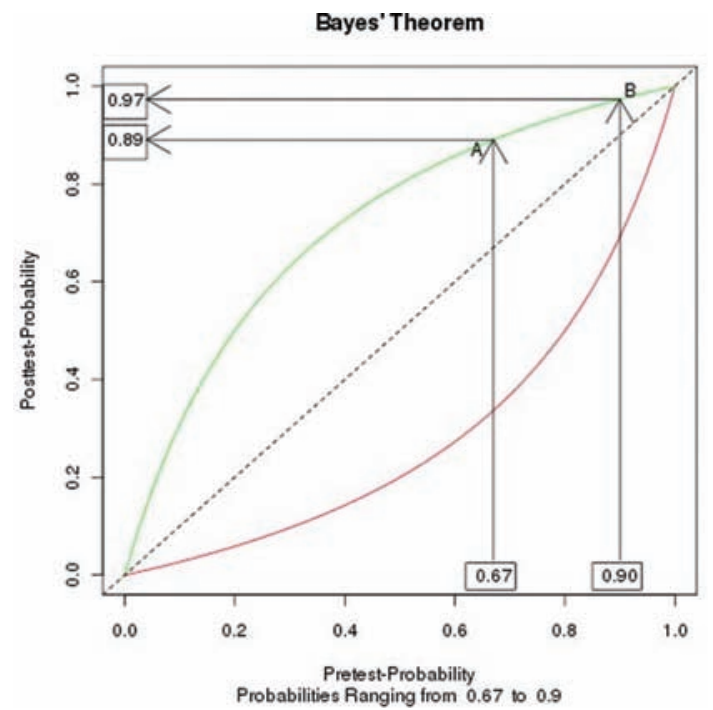

Figure 2 Graphical interpretation of Bayes' theorem for a range of pretest probabilities from $67 \%$ to $90 \%$ (likely category). 
the post-test probability (89\%) off the vertical-axis. For the upper limit of the post-test probability, one follows the arrow from a pretest probability of 90\% up until point B in figure 2, and then finds the post-test probability (97\%) on the vertical-axis. This gives the range of post-test probabilities for the likely category. For a negative result, the post-test probabilities range between 33\% and 69\%.

Next, expand the range of sensitivities and specificities from $80 \%$ to $90 \%$, representing good tests. Now, instead of a (green) curve to represent the relationship between pretest and post-test probabilities, we have a (green) band (figure 3). Like before, one follows the arrow from the pretest probability until it firsts meets the band (A in figure 3) to get the lower limit of the post-test probability and until it meets the top of the band (B in figure 3) to get the upper limit of the post-test probability. For the likely category examined above, we can see that the posttest probabilities for a positive test now range between 89\% and 99\% - almost all in the very likely category - and for a negative test between 18\% and 69\% - almost all in the uncertain or unlikely categories. The transformation of the pretest probabilities is shown as the purple inverted ' $\mathrm{L}$ ' in figure 3. The results for all the categories are shown in figure 4 and table 2.

The method is an approximation. It forces a Bayesian inspired analysis on the interpretation of test results and gives results consistent with Bayes' theorem. The approximation is weakest in the two cells with contradictory data (eg, unlikely with a positive test result and likely with a negative test result) as expected. The results remain a good approximation even with expanded ranges for test characteristics (sensitivity and specificity). For example, if the range of test characteristics is between $70 \%$ and $80 \%$, probability cut-offs of $<20 \%, 20-45 \%, 46-55 \%$, $56-80 \%$ and $>80 \%$ work well. Since the method is only an approximation and our estimates of pretest probabilities are also poor estimates, just using the cut-offs likely, uncertain and unlikely will suffice.

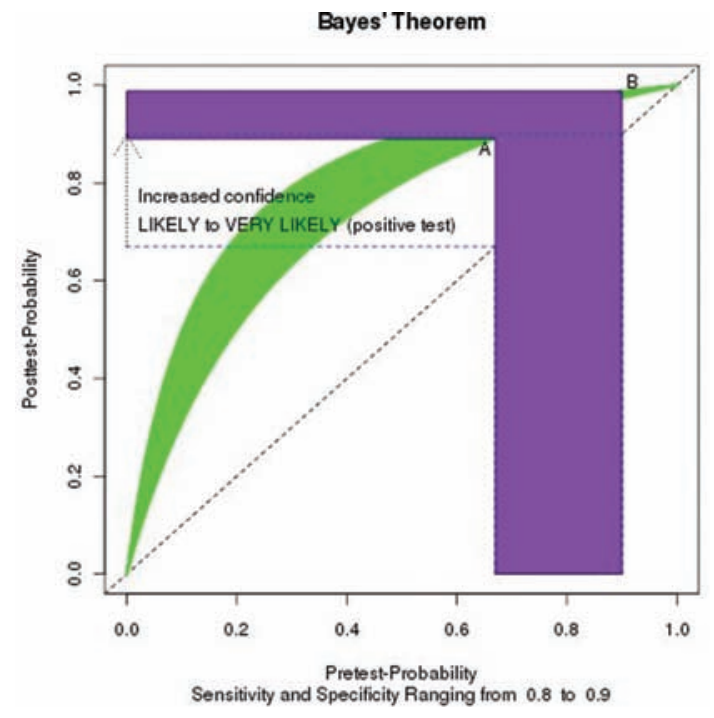

Figure 3 Graphical interpretation of Bayes' theorem for a range of pretest probabilities from $67 \%$ to $90 \%$ (likely category), and sensitivities and specificities ranging between $80 \%$ and $90 \%$.

\section{Special cases}

Rule-in and rule-out tests - tests in which a single result is capable of near definitively ruling-in or ruling-out a diagnosis - are important. For example, a low brain natriuretic peptide is suitable for ruling-out systolic heart failure. Any test with sensitivity greater than $99 \%$ is sufficient to rule-out a diagnosis from even the likely category (SnOUT) and any test with specificity greater than 99\% is sufficient to rule-in a diagnosis from even the unlikely category (SpIN).

In the very categories, since the curves are fairly flat in this region (figure 1, for example), two tests might be needed to produce a clinically significant change in the probabilities.

This method does not apply to most screening tests because the pretest probabilities are so low. The method reminds the clinician that a positive result on a screening test is usually not diagnostic, because the change in probabilities is not large enough with a single test. It will usually take two tests to go from very unlikely (as target conditions are in the general population) to very likely (the final probability a clinician is interested in before undertaking a colectomy, mastectomy or prostatectomy). For example, a 45-year-old woman has a 5-year probability of having breast cancer of about $1 \% .{ }^{11}$ The sensitivity of routine screening mammography ranges from $71 \%$ to $96 \%$ and the specificity ranges from $94 \%$ to $97 \% .{ }^{12}$ Using values of $80 \%$ for sensitivity and $96 \%$ for specificity, a positive test increases the probability to $17 \%$. Using the qualitative categories described herein, the woman's risk of breast cancer would go from very unlikely to unlikely with the single positive screening test.

Many diseases and tests have appropriate prevalences, and sensitivities and specificities for the tests published, for example, tropinin I for myocardial infarction ${ }^{13}$ or urine Chlamydia infection in men. ${ }^{14}$

\section{Summary}

In summary, here is a qualitative procedure to follow to approximate the results of a Bayesian diagnostic decision analysis.

1. What is the pretest probability of the disease being considered? Ideally this comes from an evidencebased source. If it is very likely $(<10-20 \%)$ or very unlikely (>80-90\%), in general, no further testing is needed.

2. One first categorises the pretest probability as likely, uncertain or unlikely.

3. If the test is positive, the post-test probability increases by one qualitative category (eg, unlikely to uncertain). If the test is negative, the post-test probability decreases by one qualitative category (eg, unlikely to very unlikely).

4. This process continues until the clinician is comfortable enough with the confidence in the diagnosis considering the patient's preferences, the risk of the disease and the effects of treatment.

5. Negative tests with sensitivities near 99\% can almost certainly rule out a disease, since the post-test sensitivity will be very unlikely even if the original pretest probability was likely. Similarly, positive tests with 
specificities near 99\% can almost certainly rule in a disease.

6. If the pretest probability was very likely or very unlikely, and further testing is indicated, two tests are needed to escape the very categories. This is because the change in the probabilities is small within these categories. Two concordant results are needed to change out of the very categories.
Table 2 Pretest and post-test probabilities for a categorical version of Bayes' theorem

\begin{tabular}{lllll}
\hline & \multicolumn{3}{l}{ Test result } \\
\cline { 2 - 5 } Category & Positive & \multicolumn{3}{c}{ Negative } \\
\hline Unlikely & 0.31 & 0.82 & 0.01 & 0.11 \\
Uncertain & 0.66 & 0.95 & 0.05 & 0.33 \\
Likely & 0.89 & 0.99 & 0.18 & 0.69
\end{tabular}

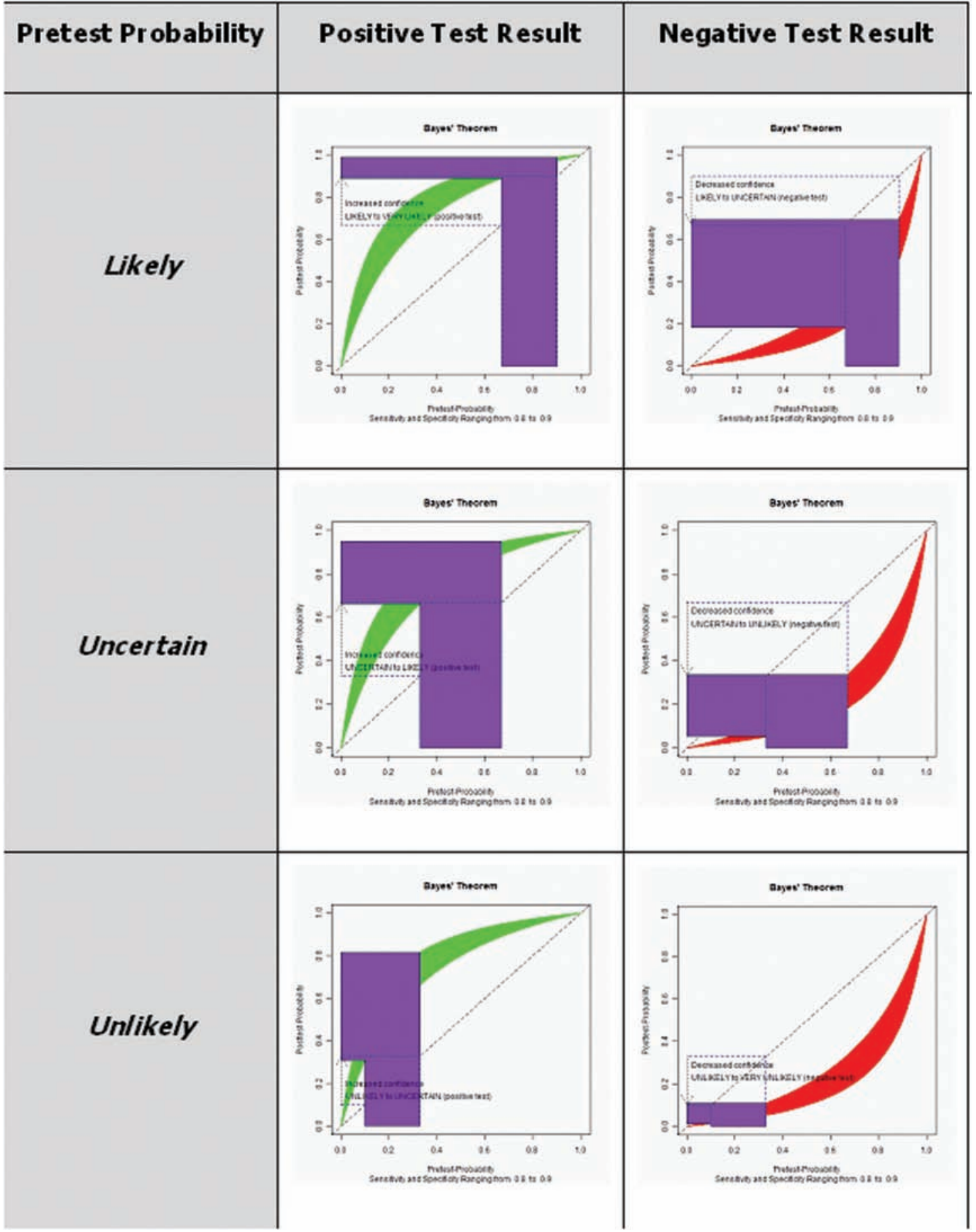

Figure 4 Graphical interpretation of Bayes' theorem for a categorical probabilities, and sensitivities and specificities between $80 \%$ and $90 \%$. 
Acknowledgments The authors would like to thank Warren Hershman, MD, MPH for his comments.

\section{Competing interests None.}

\section{References}

1. Bayes T, Price M. An Essay Towards Solving a Problem in the Doctrine of Chances. Philosophical Transactions of the Royal Society of London, 1763:370-418.

2. Horrocks JC, McCann AP, Staniland JR, et al. Computer-aided diagnosis: description of an adaptable system, and operational experience with 2,034 cases. Br Med J 1972;2:5-9.

3. Friedman CP, Elstein AS, Wolf FM, et al. Enhancement of clinicians' diagnostic reasoning by computer-based consultation: a multisite study of 2 systems. JAMA 1999;282:1851-6.

4. Fagan TJ. Letter: nomogram for Bayes theorem. N Engl J Med 1975;293:257.

5. Hellmich M, Lehmacher W. A ruler for interpreting diagnostic test results. Methods Inf Med 2005;44:124-6.

6. Schwartz A. Diagnostic Test Calculator (Version 2010042101). http://araw.mede.uic.edu/cgi-bin/testcalc.pl (accessed 2 February 2011).

7. Elstein AS, Schwartz A, Schwarz A. Clinical problem solving and diagnostic decision making: selective review of the cognitive literature. BMJ 2002;324:729-32.
8. Mark DB. Decision-making in clinical medicine. In: Fauci AS, Braunwald E, Kasper DL, Hauser SL, Longo DL, Jameson JL, Loscalzo J, eds. Harrison's Principles of Internal Medicine. 17th edition. Harrison's, NY: McGraw-Hill Professional 2008:2958.

9. Pauker SG, Kassirer JP. The threshold approach to clinical decision making. N Engl J Med 1980;302:1109-17.

10. Diamond GA, Forrester JS. Analysis of probability as an aid in the clinical diagnosis of coronary-artery disease. $N$ Engl J Med 1979;300:1350-8.

11. National Cancer Institute. Breast Cancer Risk Assessment Tool. http://www.cancer.gov/bcrisktool/RiskAssessment. aspx? current_age $=45 \mathrm{Ctage}$ _at_menarche=998tage_at_first_live_ birth=99\&tever_had_biopsy=99\&previous_biopsies=99\&tbiopsy_ with_hyperplasia=99\&trelated_with_breast_cancer=99\&trace $=1$ (accessed 23 March 2011).

12. Cheng T, Freund KM, Lane S, et al. Screening for Breast Cancer. ACP PIER: The Physicians' Information and Education Resource. http://pier.acponline.org/physicians/screening/s369/ effectiveness/s369-s3.html (accessed 23 March 2011).

13. Keller T, Zeller T, Peetz D, et al. Sensitive troponin I assay in early diagnosis of acute myocardial infarction. $N$ Engl J Med 2009;361:868-77.

14. Nadala EC, Goh BT, Magbanua JP, et al. Performance evaluation of a new rapid urine test for chlamydia in men: prospective cohort study. BMJ 2009;339:b2655. 